\title{
EFEITO DO CARBARYL SOBRE O 'RUSSETING' DA MAÇÃ (MALUS DOMESTICA BORKH.), CULTIVARES 'GALA', 'FUJI' E 'GOLDEN DELICIOUS'
}

\author{
ANÍSIO PEDRO CAMILO², FREDERICO DENARDI ${ }^{3}$
}

\begin{abstract}
RESUMO - O "russeting" da maçã caracteriza-se por uma camada de cortiça formada entre as células da epiderme e que dá um aspecto de rugosidade à superfície do fruto, depreciando-o para a comercialização. O raleio de frutos é uma prática cultural bastante difundida entre os produtores de maçã. Pode ser efetuada manualmente, quimicamente ou pela associação de ambos. Dentre os produtos mais usados para raleio químico, estão o ácido naftaleno acético (ANA) e o carbaryl, um inseticida carbamato, conhecido comercialmente como Sevinâ. Há duas formulações de carbaryl no mercado brasileiro, mas não existem dados de pesquisa suficientes que permitam escolher a formulação mais adequada. Há citações de que o carbaryl pode causar "russeting" em maçãs. O objetivo deste trabalho foi testar as duas formulações de carbaryl existentes no mercado, quanto ao seu efeito sobre o "russeting" nas maçãs. Os experimentos foram conduzidos em Fraiburgo e em Caçador, Santa Catarina, Brasil. Foram avaliadas duas concentrações, 500 ppm e 1.500 ppm de carbaryl. Foram testadas duas formulações, uma em pó-molhável com $85 \%$ de i.a. e outra em suspensão concentrada com $48 \%$ de i.a. Como a incidência de "russeting" varia entre cultivares, testou-se em 'Gala', 'Fuji' e 'Golden Delicious', que são as três mais importantes no mercado brasileiro. Os resultados mostraram que: 1) A ocorrência de "russeting" para as três cultivares foi maior em Fraiburgo do que em Caçador; 2) O carbaryl, na formulação solução concentrada, causou mais "russeting" em 'Golden Delicious', em Caçador, indicando que o seu uso deve ser evitado para essa cultivar, dando-se preferência à formulação pó-molhável; 3)Tanto a formulação quanto a concentração de carbaryl não afetaram a incidência de "russeting” nas cultivares 'Gala' e 'Fuji' nos dois locais.
\end{abstract}

Termos para indexação: “russeting”, raleio químico, carbaryl, ácido naftaleno acético, maçã.

\section{EFFECT OF CARBARYL ON RUSSETING OF APPLE (Malus domestica Borkh.), CULTIVARS 'GALA', 'FUJI' AND 'GOLDEN DELICIOUS'}

\begin{abstract}
Russeting is characterized by the development of a cork layer among the epidermal cells giving an aspect of rugosity to the fruit surface, reducing its marketability. Fruit thinning is a technique widely used by apple growers. It can be done by hand or by using chemical thinning or by both, chemical and hand thinning. Among the most used chemical for thinning are naphthaleneacetic acid (NAA) and the insecticide carbaryl, commonly known as Sevinâ. In the Brazilian market there are two formulations of carbaryl: wetted powder and flowable solution. None of them are registered as chemical thinning for apple in Brazil. However there is not enough official research data in Brazil in this area that could support the best choice as far as russeting on the fruit is concerned. Some authors say that carbaryl may cause russeting on apples. This trial aimed to evaluate the two carbaryl formulations comparing its effect concerned to russeting. The experiments were carried out in two areas: Fraiburgo and Caçador, Santa Catarina State, Brazil. Two concentrations: $500 \mathrm{ppm}$ and $1.500 \mathrm{ppm}$ of each formulation were tested. One of the formulations was a wetted powder with $85 \%$ of a.i., the other was a flowable solution with $48 \%$ of a.i. As the incidence of russeting on apple may vary according to cultivar, the experiments were carried out on 'Gala', 'Fuji' and 'Golden Delicious'. It was concluded that: 1) Fraiburgo climactic conditions were much more favorable to russeting than those in Caçador; 2) carbaryl did not cause russeting on 'Gala' and 'Fuji' in both locations; 3) Carbaryl as flowable solution caused more russeting in 'Golden Delicious' at Caçador, so this formulation should not be recommended for this cultivar.
\end{abstract}

Index terms: russeting, chemical thinning, carbaryl, naphthaleneacetic acid, apple.

\section{INTRODUÇÃO}

O carbaryl, usado tanto como inseticida quanto como raleante de frutos, pode induzir "russeting" em maçãs (Stubbings \& Strydom, 1965; Myburgh \& Heyns, 1966).

A presença de "russeting" deprecia comercialmente as maçãs e pode restringir seriamente sua aceitação, especialmente no mercado externo. No Chile, segundo Gonzalo (1989), o "russeting" pode causar um descarte de frutos para exportação da ordem de 20 a $30 \%$ na cultivar 'Delicious' e de 10 a $15 \%$ na 'Granny Smith'. Segundo o mesmo autor, a cultivar 'Golden Delicious' chilena ficou inviabilizada para exportação devido sua alta suscetibilidade ao "russeting". Na África do Sul, anualmente, grande parte da produção de 'Golden Delicious' não é exportada devido à presença de "russeting" (Damas, 1989).

O "russeting" da maçã é caracterizado pela presença de uma camada de cortiça formada nas células da epiderme. A causa primária de sua formação são fatores externos à planta que levam

1 (Trabalho 006/2001). Recebido: 08/01/2001. Aceito para publicação: 30/08/2001.

2 Eng. Agr., Ph.D. em Pomologia e Melhoramento Genético, EMBRAPA/EPAGRI-Estação Experimental de Caçador, C. P. 591, Fone 0xx49 5630211, Fax 0xx19 5633211, 89500-000, Caçador, SC, Brasil. e-mail: camilo@epagri.rct-sc.br.

3 Eng. Agr. M. Sc. em Fruticultura de Clima Temperado, EPAGRI-Estação Experimental de Caçador, C. P. 591, Fone 0xx49 563 0211, FAX 0xx49 563 3211, 89500-000, Caçador, SC, Brasil. e-mail: denardi@epagri.rct-sc.br 
à formação de fendas na cutícula que envolve o fruto, expondo as células que ficam logo abaixo desta camada. Sob condições de alta umidade relativa, aquelas células sofrem danos, e a conseqüente reação protetora da planta é isolar as áreas danificadas através da formação deste tecido de cortiça (Tukey, 1969; Steenkamp et al., 1984).

Embora o "russeting" possa ocorrer a qualquer momento no desenvolvimento dos frutos, estes são mais suscetíveis no estádio inicial de seu desenvolvimento (Gonzalo, 1989), sendo primariamente dependente de condições climáticas favoráveis. Poderá ser reduzido ao mínimo se os frutos forem ensacados a partir do estádio inicial de seu desenvolvimento (Camilo et al., 1976; Creazy \& Swartz, 1981).

Durante o período crítico favorável ao "russeting" (início de desenvolvimento dos frutos), produtos químicos, tais como enxofre, oxicloreto de cobre, quelato de ferro, dodine, e organofosforados como parathion, malathion, ethion, guthion e diazinon, se aplicados sob condições de baixa temperatura e de alta umidade relativa (baixa evaporação), podem induzir a formação de "russeting" (Stubbings \& Strydom, 1965; Slade, 1979).

Produtos na formulação emulsionável são mais prováveis causadores de "russeting" do que os na formulação pó-molhável. Quando aplicados sob temperaturas elevadas, os perigos de incidência de "russeting" são ainda mais graves devido à maior penetração através da cutícula que envolve o fruto (Gonzalo, 1989).

Este trabalho teve como objetivo verificar o comportamento do carbaryl nas formulações de Pó-Molhável (PM) e Solução Concentrada (SC) sobre a incidência de "russeting" no fruto das cultivares 'Gala', 'Golden Delicious' e 'Fuji', nas condições de Fraiburgo e de Caçador, Santa Catarina.

\section{MATERIAIS E MÉTODOS}

Os experimentos foram conduzidos durante o ciclo 1994/ 95, em pomar da empresa Renar Maçãs S/A, em Fraiburgo-SC, e em pomar da EPAGRI/Estação Experimental de Caçador, município de Caçador-SC, ambos com altitude ao redor dos $1.100 \mathrm{~m}$ acima do nível do mar. As cultivares testadas foram 'Fuji', 'Gala' e 'Golden Delicious' sobre o porta-enxerto M7, plantio de 1982 (Fraiburgo) e 1978 (Caçador). Os espaçamentos das plantas foram de $5,0 \mathrm{~m} \times 2,5 \mathrm{~m}$ (Fraiburgo) e $5,0 \mathrm{~m} \times 3,0 \mathrm{~m}$ (Caçador) para as três cultivares.

O delineamento experimental foi em blocos ao acaso, com cinco tratamentos e seis repetições de uma planta por parcela. Foi deixada uma planta como bordadura entre duas plantas úteis e uma fila de planta como bordadura entre duas filas de plantas úteis. Em todos os tratamentos envolvendo raleantes químicos, adicionou-se $0,2 \%$ de um óleo mineral emulsionável como adjuvante, conforme a recomendação de raleio químico do Sistema de Produção para a Cultura da Macieira da Empasc/Emater (1991).

Os produtos usados foram Sevin ${ }^{\circledR} 850 \mathrm{PM}$ (pó-molhável), com $85 \%$ de ingrediente ativo, e Sevin ${ }^{\circledR} 480$ SC (solução concentrada), com $48 \%$ de ingrediente ativo (Aventis Crop Science Brasil Ltda., Av.Maria Coelho Aguiar, 215, Bloco B - 20 Andar, São Paulo-SP, Cep 05804-902)

Os tratamentos testados foram os seguintes: 1. Ácido Naftaleno Acético (ANA) a 7,5 ppm aos 5 dias após a plena floração (DAPF) + Sevin ${ }^{\circledast} 850$ PM a 500 ppm aos 15 DAPF; 2. ANA a 7,5 ppm aos 5 DAPF + Sevinâ 480 SC a 500 ppm aos 15 DAPF; 3. ANA a 7,5 ppm aos 5 DAPF + Sevinâ 850 PM a 1.500 ppm aos 15 DAPF; 4. ANA a 7,5 ppm aos 5 DAPF + Sevinâ 480 $\mathrm{SC}$ a 1.500 ppm aos $15 \mathrm{DAPF} ; 5$. Raleio manual, deixando-se 2,1 ou nenhum fruto por gema, de acordo com o vigor das gemas de frutificação, efetuado aos $30 \mathrm{DAPF}$. Os tratamentos com raleantes químicos foram aplicados com pulverizador manual estacionário, com pistola de cabo longo e bico Yamaho HV-3. As plantas foram molhadas até próximo do ponto de gotejamento. $\mathrm{O}$ volume médio de calda gasto variou entre 2,0 e 2,5L/planta, dependendo do volume da copa.

Cem frutos por repetição foram amostrados ao acaso ao redor da planta, na colheita, para avaliar o efeito dos tratamentos quanto à incidência de "russeting". Os frutos foram classificados em cinco classes, segundo a incidência de "russeting", baseada em observação visual nas cinco classes descritas abaixo.

Classe 1: ausência de "russeting", sendo tolerado somente aquele restrito à cavidade peduncular; Classe 2: presença de "russeting" não superando $10 \%$ da superfície do fruto; Classe 3 : incidência de "russeting" entre $10 \%$ e $30 \%$ da superfície do fruto; Classe 4 : incidência de "russeting" entre $30 \%$ e $50 \%$ da superfície do fruto; Classe 5: incidência de "russeting" superior a 50\% da superfície do fruto.

As médias foram comparadas pelo teste de Duncan, com o auxílio da fórmula de Townsend \& Heuberger (1943), que leva em consideração as cinco classes de incidência de "russeting". As datas de plena floração, época de aplicação dos tratamentos e época de colheita constam nas Tabelas 1 e 2 para Fraiburgo e Caçador, respectivamente.

\section{RESULTADOS E DISCUSSÃO}

Os resultados mostraram que a incidência de "russeting" foi diferente nos dois locais testados, ficando evidenciado que a ocorrência desse problema em Fraiburgo foi muito mais grave que em Caçador (Tabela 3). Isto sugere que fatores climáticos e, possivelmente, o emprego intensivo de certos produtos químicos, usados para o controle de pragas e de doenças, possam estar mais envolvidos na ocorrência de "russeting" do que uma única aplicação de carbaryl utilizada como raleante. O efeito do local sobre a incidência de "russeting" foi também observado por Wertheim (1986). Creazy \& Swartz (1981) observaram que, embora a quantidade de "russeting" em um específico local tenha variado de ano para ano, em resposta às condições climáticas, determinados locais sempre apresentaram mais "russeting" do que outros.

A 'Golden Delicious' foi a que mostrou maior incidência de "russeting" dentre as três cultivares testadas (Tabela 3 ). Bleicher (1993), baseado em uma série de experimentos, também constatou que esta é a mais suscetível dentre as três principais cultivares de macieira plantadas no Sul do Brasil.

Os resultados indicaram que, para as cultivares 'Gala' e 'Fuji', a incidência de "russeting" não está relacionada ao uso do ANA associado ao carbaryl como raleantes (Tabela 4). Estas observações confirmam resultados de Camilo et al. (1991), que também não detectaram efeito do ANA associado ao carbaryl na incidência de "russeting" na cultivar 'Gala', mostrando que existe 
TABELA 1 - Época de plena floração, aplicação dos tratamentos e de colheita das cultivares 'Gala', 'Golden Delicious' e 'Fuji' . Fraiburgo-SC, ciclo 1994/1995.

\begin{tabular}{lccc}
\hline Evento & 'G a la' & 'G.Delicious & 'F uji \\
\hline Plena floração & $10-10-94$ & $10-10-94$ & $12-10-94$ \\
A plicação do A N A & $15-10-94$ & $15-10-94$ & $17-10-94$ \\
A plicação carbaryl & $27-10-94$ & $27-10-94$ & $27-10-94$ \\
R aleio manual & $10-11-94$ & $10-11-94$ & $12-11-94$ \\
Colheita & $15-02-95$ & $23-02-95$ & $29-03-95$ \\
\hline
\end{tabular}

TABELA 2 - Época de plena floração, aplicação dos tratamentos e de colheita das cultivares 'Gala', 'Golden Delicious' e 'Fuji'. Caçador-SC, ciclo 1994/1995.

\begin{tabular}{lccc}
\hline Even to & G a la & G.Delicious & F uji \\
\hline P lena floração & $12-10-94$ & $18-10-94$ & $07-10-94$ \\
A plicação do A N A & $19-10-94$ & $25-10-94$ & $12-10-94$ \\
A plicação carbaryl & $28-10-94$ & $03-11-94$ & $22-10-94$ \\
Raleio manual & $12-11-94$ & $18-11-94$ & $07-11-94$ \\
Colheita & $16-02-95$ & $25-02-95$ & $30-03-95$ \\
\hline
\end{tabular}

TABELA 3 - Efeito de local sobre a incidência de 'russeting' no fruto nas cultivares 'Gala', 'Golden Delicious' e ‘Fuji', ciclo $1994 / 1995$.

\begin{tabular}{|c|c|c|}
\hline \multirow[t]{2}{*}{ Cultivar } & \multicolumn{2}{|c|}{ Incidência de "Russeting"/ Local ${ }^{1}$} \\
\hline & Fraiburgo & Caçador \\
\hline 'G a la' & $34,8 \mathrm{~A}$ & $30,7 \mathrm{~B}$ \\
\hline 'Golden Delicious' & $63,9 \mathrm{~A}$ & $38,5 \mathrm{~B}$ \\
\hline
\end{tabular}

${ }^{1}$ Médias seguidas pela mesma letra, na mesma linha, não diferem entre si, pelo teste de Duncan, ao nível de 5\% de probabilidade.

TABELA 4 - Efeito dos tratamentos de raleio sobre a percentagem de “russeting” nas cultivares 'Gala', 'Fuji' e 'Golden Delicious' em Fraiburgo e em Caçador-SC, ciclo 1994/1995.

\begin{tabular}{|c|c|c|c|c|c|c|}
\hline \multirow{3}{*}{ Tratamentos } & \multicolumn{6}{|c|}{ Percentagem de "Russeting" no Fruto ${ }^{1}$} \\
\hline & \multicolumn{2}{|c|}{ Ga la'، } & \multicolumn{2}{|c|}{ 'F uji' } & \multicolumn{2}{|c|}{ 'Golden Delicious' } \\
\hline & & --- & & & & \\
\hline $\begin{array}{l}\text { 1. ANA } 7.5 \mathrm{ppm}+\text { Sevin } \\
850 \text { PM } 500 \mathrm{ppm}\end{array}$ & 33,2 a & $30,8 \mathrm{a}$ & $34,6 \mathrm{a}$ & $27,6 \mathrm{a}$ & $62,8 \mathrm{a}$ & $34,2 \mathrm{bc}$ \\
\hline $\begin{array}{l}\text { 3. ANA } 7.5 \mathrm{ppm}+\text { Sevin } \\
850 \mathrm{PM} 1500 \mathrm{ppm}\end{array}$ & 35,2 a & 30,8 a & 36,9 a & 25,9 a & 67,8 a & $40,7 \mathrm{ab}$ \\
\hline $\begin{array}{l}\text { 4. ANA } 7.5 \text { ppm }+ \text { Sevin }^{\circledR} \\
480 \text { SC } 1500 \text { ppm }\end{array}$ & 38,5 a & $29,1 \mathrm{a}$ & 34,5 a & $26,9 \mathrm{a}$ & $67,2 \mathrm{a}$ & 43,6 a \\
\hline $\mathrm{CV}(\%)$ & 14,3 & 11,1 & 11,9 & 20,0 & 9,7 & 14,6 \\
\hline
\end{tabular}

1 Médias seguidas pela mesma letra, dentro de cada coluna, não diferem entre si, pelo teste de Duncan, ao nível de $5 \%$ de probabilidade. NS Não significativo ao nível de $5 \%$ de probabilidade.

** Significativo ao nível de $1 \%$ de probabilidade. 
diferença varietal quanto à suscetibilidade ao "russeting" da maçã.

O carbaryl, em solução concentrada, induziu maior incidência de "russeting" na cultivar Golden Delicious em Caçador, comparado à formulação pó-molhável (Tabela 4). Com base nesta observação, a formulação solução concentrada de carbaryl deve ser usada com cautela na cultivar 'Golden Delicious' e, por extensão, nas outras cultivares do mesmo grupo ('Belgolden', 'Golden Spur', 'Smoothe', e outras). Este efeito do carbaryl em 'Golden Delicious' está de acordo com as observações de Gonzalo (1989), que salientou que produtos na formulação solução concentrada podem promover mais "russeting" do que os na formulação pó-molhável, especialmente quando aplicados com temperaturas altas. A temperatura máxima $\left(27^{\circ} \mathrm{C}\right)$, registrada no dia da aplicação do carbaryl em Caçador, foi mais alta do que aquela em Fraiburgo $\left(23,4^{\circ} \mathrm{C}\right)$. Gonzalo (1989) também explica que a formulação solução concentrada possui maior capacidade de penetração através da cutícula, possibilitando o contato com as membranas das células da epiderme, promovendo a reação destas com a conseqüente formação de "russeting".

\section{CONCLUSÕES}

1. A ocorrência de "russeting" foi maior em Fraiburgo para as três cultivares testadas.

2. O carbaryl, na formulação solução concentrada, causou mais “russeting” em frutos de 'Golden Delicious' em Caçador e, quando possível, deve ser evitado para essa cultivar, dando-se preferência para a formulação pó-molhável na concentração de $500 \mathrm{ppm}$.

3. Tanto a formulação quanto a concentração de carbaryl não afetaram a incidência de "russeting" nas cultivares 'Gala' e 'Fuji', em ambos os locais.

\section{REFERÊNCIAS BIBLIOGRÁFICAS}

BLEICHER, J. O 'russeting' na cultura da macieira. In: RELATÓRIO dos Projetos do Convênio EPAGRI/Estação Experimental de Caçador - ABPM, 1992-93. Caçador: EPAGRI, 1993.p.68-81.

CAMILO, A.P; MONDARDO, M.; LEITE, G. B. Raleio de frutos de macieira, cultivar Gala. Revista Brasileira de Fruticultura, Cruz das Almas, BA, v.13, n.1, p.145-152, 1991.

CAMILO, A.P.; PETRI, J.L.; CAMELATTO, D. Controle do 'russeting' da macieira Golden Delicious (Malus domestica,
Borkh.) por meio de ensacamento dos frutos. In: CONGRESSO BRASILEIRO DE FRUTICULTURA, 3., 1975, Rio de Janeiro. Anais: Campinas: Sociedade Brasileira de Fruticultura, 1976, v.2, p.461-465.

CREAZY, L.L.; SWARTZ, H.J. Agents influencing russeting on Golden Delicious apple fruits. Journal of American Society for Horticultural Science, Alexandria, v.106, n.2, p.203-206, 1981.

DAMAS, H.F. Russeting of 'Golden Delicious' apples. The Deciduous Fruit Grower, Bellville, v.39, n.11, p.421-425, 1989.

EMPASC/ACARESC. Sistema de Produção para a cultura da macieira. 3.rev. Florianópolis, 1991. 71 p. (Sistema de Produção, 19).

GONZALO, F.G.S. La rugosidad o ruginosidad ("russet") de la fruta. Revista Fruticola, Curicó, Chile, v.10, n.2, p.57-62, 1989.

MYBURGH, A.C.; HEYNS, A.D. The inducement of russeting by pesticides on 'Golden Delicious' apples. The Deciduous Fruit Grower, Bellville, v.16, p.314-318, 1966.

SLADE, D.A. Russeting of apples - A review of work undertaken at Massey University. Orchadist

of New Zealand, Wellington, v.52, n.2, p.58-59, 1979.

STEENKAMP, J.; VAN ZYL, H.J.; WESTRAAD, I. A preliminary evaluation of various chemical substances for the control of calyx-end russeting in 'Golden Delicious'. Journal of Horticultural Science, Maidstone, v.59, n.4, p.501-505, 1984

STUBBINGS, W.A.K.; STRYDOM, D.K. Russeting of Golden Delicious apples in Elgin area. The Deciduous Fruit Grower, Bellville, v.15, p.149-151, 1965.

TOWNSEND, G.R.; HEUBERGER, J.W. Methods for estimating losses caused by diseases in fungicides experiments. The Plant Diseases Reporter, Washington, v.27, n.17, p.340-343, 1943.

TUKEY, L.D. Observation on the russeting of apples growing in plastic bags. Proceedings of the American Society for Horticultural Science, Alexandria, v.74, p.30-39, 1969.

WERTHEIM, S.J. Chemical thinning of Golden Delicious apple with NAAm and/or carbaryl in combination with a spreader and the anti-russeting agent $\mathrm{GA}_{4+7}$. Acta Horticulturae, Wageningenn. 179, p. 659-66, 1986. 